Une expérience de transfert de résultats de recherche : évaluation de Clicfrançais, des scénarios pédagogiques en ligne destinés à l'insertion socioprofessionnelle An study of transfer of research results : evaluation of Clicfrançais, on-line teaching scenarios intended for socio-professional insertion Una experiencia de transferencia de resultados de investigación : evaluación de Clicfrançais, situaciones pedagógicas en línea destinadas a la inserción socioprofesional

Godelieve Debeurme et Isabelle Landry

\section{Volume 35, numéro 1, 2009}

Avoir des difficultés scolaires importantes à l'école : quelles formules, quel avenir?

URI : https://id.erudit.org/iderudit/029924ar

DOI : https://doi.org/10.7202/029924ar

Aller au sommaire du numéro

Éditeur(s)

Revue des sciences de l'éducation

ISSN

0318-479X (imprimé)

1705-0065 (numérique)

Découvrir la revue

Citer cet article

Debeurme, G. \& Landry, I. (2009). Une expérience de transfert de résultats de recherche : évaluation de Clicfrançais, des scénarios pédagogiques en ligne destinés à l'insertion socioprofessionnelle. Revue des sciences de l'éducation, 35(1), 69-98. https://doi.org/10.7202/029924ar

\section{Résumé de l'article}

Depuis plusieurs années, les élèves présentant des difficultés d'acquisition de la langue font partie de nos préoccupations. Pour intervenir auprès d'eux, il est primordial de les placer dans des contextes d'apprentissage motivants et d'offrir des activités adaptées à leurs besoins, voire à leurs capacités. C'est dans ce but que Clicfrançais a été élaboré dans le cadre d'un transfert de connaissances financé par le Centre de transfert pour la réussite éducative du Québec (CTREQ). Dans cet article, nous présentons le chemin parcouru entre des résultats de recherche et leur aboutissement en un outil didactique en ligne, mis à la disposition des jeunes en insertion socioprofessionnelle. Nous décrivons le contenu de l'outil et rapportons les résultats de son évaluation partielle par un nombre restreint d'élèves et d'enseignants. 


\title{
Une expérience de transfert de résultats de recherche: évaluation de Clicfrançais, des scénarios pédagogiques en ligne destinés à l'insertion socioprofessionnelle ${ }^{1}$
}

\author{
Godelieve Debeurme, professeure \\ Université de Sherbrooke
}

Isabelle Landry, chargée de projets Centre de transfert pour la réussite éducative du Québec (CTREQ)

\begin{abstract}
RÉSUMÉ - Depuis plusieurs années, les élèves présentant des difficultés d'acquisition de la langue font partie de nos préoccupations. Pour intervenir auprès d'eux, il est primordial de les placer dans des contextes d'apprentissage motivants et d'offrir des activités adaptées à leurs besoins, voire à leurs capacités. C'est dans ce but que Clicfrançais a été élaboré dans le cadre d'un transfert de connaissances financé par le Centre de transfert pour la réussite éducative du Québec (CTREQ). Dans cet article, nous présentons le chemin parcouru entre des résultats de recherche et leur aboutissement en un outil didactique en ligne, mis à la disposition des jeunes en insertion socioprofessionnelle. Nous décrivons le contenu de l'outil et rapportons les résultats de son évaluation partielle par un nombre restreint d'élèves et d'enseignants.
\end{abstract}

MOTS CLÉS • insertion socioprofessionnelle, difficultés langagières, enseignement secondaire, outil en ligne, transfert de connaissances.

\section{Introduction et problématique}

\section{1 État de la situation en cheminement particulier de formation}

Alors que de nombreuses instances exigent un minimum de qualification professionnelle et un diplôme d'études secondaires comme seuil minimal de formation nécessaire à l'insertion sociale et professionnelle, beaucoup de jeunes ( $32 \%$ selon Savoie-Zajc et Lanaris, 2005, p. 297) quittent chaque année l'école avant d'avoir terminé un programme d'études secondaires, manifestant de multiples lacunes en éducation de base. Parmi ces décrocheurs se trouvent un bon nombre d'élèves éprouvant des difficultés d'apprentissage ou de comportement, qui ont entrainé des retards scolaires de plus en plus criants, accompagnés d'un sentiment de

1. Nous remercions toutes les personnes qui se sont prêtées à la pré-expérimentation et à l'évaluation du site. 
démotivation et d'une perception négative d'eux-mêmes. Pour plusieurs chercheurs (Baby, 2005; Bak, 1991; Parent et Parent, 1994: voir Debeurme, 1999; Vultur, 2005), ces jeunes sont généralement des élèves qui présentent des besoins différents, et auxquels les matières scolaires, les pratiques pédagogiques et les conditions d'apprentissage de l'école, bref la tournure d'esprit de l'école, pour citer Baby (2005), ne répondent pas.

Au fil du temps, des programmes adaptés visant à favoriser l'insertion socioprofessionnelle de ces élèves ont été mis en place au Québec afin de répondre davantage à leurs besoins et de contribuer à leur réussite dans la société. Ainsi sont apparus les programmes de cheminement particulier, temporaire et continu, d'insertion sociale et professionnelle des jeunes (ISPJ), d'alternance études-travail (AET), de même que les Centres de formation en entreprise et récupération (CFER). Liés au secteur des jeunes, ces programmes sont destinés à des élèves âgés de 15 à 21 ans.

Avant d'y participer, la majorité d'entre eux ont vécu un cumul d'échecs causés souvent par des difficultés en lecture et en écriture, fréquemment sources de leur retard scolaire dès les premières années. Par ailleurs, rappelons que, dans notre société du savoir, gérée en bonne partie par les TIC et exigeant un traitement constant d'informations simples et complexes, $11 \%$ des jeunes de 16 à 25 ans sont classés, sur une échelle de compétence, au niveau le plus faible, et à $26 \%$ de la proportion au niveau 2, niveaux jugés insuffisants pour s'intégrer dans la société de l'écrit (Bourdon et Roy, 2005). Leur insertion n'en sera certes pas facilitée. Si on a constaté régulièrement un manque d'investissement dans l'apprentissage du français, c'est aussi parce que ces jeunes ne lui ont trouvé ni sens ni utilité. En effet, selon ces derniers, les situations d'apprentissage sont trop répétitives, pas assez concrètes et les préparent mal à l'intégration au monde du travail et à ses exigences (Debeurme, 1995).

Par surcroît, les maisons d'édition québécoises ont développé très peu, sinon pas, de matériel didactique adapté aux capacités et aux intérêts spécifiques de ces élèves. D'ailleurs, le Rapport sur l'évaluation du cheminement particulier de formation visant l'insertion sociale et professionnelle des jeunes de 16 à 18 ans (ISPJ) (Ministère de l'Éducation du Québec, 2003) faisait état, en termes de soutien, aux équipes, de nombreux besoins d'intervention, d'adaptation de matériel, de création d'outils pédagogiques appropriés aux élèves avec un cheminement particulier, partie intégrante de la mise en place des conditions favorisant la réussite des élèves. Cette absence de matériel adapté a souvent obligé les enseignants et formateurs à recourir aux modules de l'enseignement individualisé, que l'on sait peu d'actualité, ou à construire leur propre matériel, ce qui exigeait beaucoup de temps et d'investissement de leur part. Rappelons que nombre de ces enseignants se retrouvent dans ces programmes malgré eux, et préferent alors s'appuyer sur une pédagogie conventionnelle (Baby, 2005, p. 22) en puisant dans du matériel destiné aux classes ordinaires, avec le risque d'entraîner rapidement une perte d'intérêt chez leurs élèves. 
Toutefois, malgré ces lacunes, il ne faut pas passer sous silence les multiples initiatives individuelles prises par de nombreux enseignants. Ainsi, citons en exemple les programmes des Centres de formation en entreprise et récupération, où l'on a choisi d'utiliser les journaux comme soutiens à l'apprentissage du français, ce qui permet d'assurer non seulement le développement des compétences en français, mais aussi une ouverture sur le monde, un des objectifs prioritaires pour former un citoyen engagé.

\subsection{Des conclusions théoriques aux scénarios d'apprentissage adaptés}

Sensibilisées par cette problématique et soucieuse, de mettre à profit les conclusions tirées d'une expérimentation antérieure, nous avons accompli un projet de transfert de connaissances, initié et soutenu par le Centre de Transfert pour la Réussite Éducative du Québec (CTREQ).

L'outil didactique réalisé est issu de l'expérimentation d'activités d'apprentissage créées pour les fins de la thèse doctorale et ayant comme cadre de référence les principes de l'éducation à la carrière. En effet, selon Hoyt (1987, p. 34):

L'éducation à la carrière est un effort de collaboration entre le système de l'éducation et

l'ensemble de la communauté qui a pour but de réformer l'éducation, principalement par

l'infusion de notions liées à la carrière dans des activités scolaires, à relier l'éducation et le travail et à acquérir des habiletés générales d'employabilité [...].

L'éducation à la carrière est une source d'inspiration pour l'approche orientante dans le Renouveau pédagogique du ministère de l'Éducation, du Loisir et du Sport, basé sur le modèle de dynamique motivationnelle de Viau (1994) et d'une approche psycholinguistique en enseignement de la langue maternelle. Le Comité sur l'approche orientante en Estrie définit celle-ci en ces termes:

Le concept de l'approche orientante intègre l'information et l'orientation scolaires et professionnelles à l'ensemble des activités de l'école de façon concertée et en collaboration avec tous les intervenants de l'école, les parents et la communauté. S'appuyant sur les phases du processus d'orientation, l'approche orientante vise à favoriser la motivation et l'engagement de l'élève dans son cheminement individuel en lien avec ses choix scolaires et professionnels (2002, p. 5).

L'adaptation des scénarios aux besoins d'une population scolaire du secondaire maîtrisant peu de compétences lecturales et scripturales, a été guidée par des pistes tirées des écrits de recherche, par les résultats d'un processus de consultations auprès d'enseignants et grâce aux observations des stagiaires en cours de formation.

En guise de pré-expérimentation, une évaluation du matériel a été réalisée auprès d'une dizaine d'enseignants des programmes adaptés dans des écoles de la Montérégie et de l'Estrie. Une première collecte a été effectuée à l'aide d'une grille critériée couvrant six aspects: 1) la pertinence, 2) l'utilité, 3) la faisabilité, 4) le degré d'adaptation, 5) l'originalité et 6) les limites des activités (voir l'annexe). Les réponses ont été 
analysées par une étudiante en orientation scolaire et professionnelle dans le cadre d'un essai de maîtrise (Plouffe, 2000); des échanges ont eu lieu avec d'autres enseignants sur les besoins de matériel didactique. Par la suite, un document validé, regroupant une trentaine de scénarios d'apprentissage conformes aux exigences ministérielles du programme-cadre d'insertion sociale et professionnelle des jeunes, a été présenté en appel d'offres au Centre de transfert pour la réussite éducative du Québec, dont la mission est d'accroître la réussite éducative par la production, la diffusion et l'accompagnement d'instruments d'intervention adaptés aux utilisateurs en s'appuyant sur le transfert de résultats de recherches. Ainsi donc, ce sont des écrits et des résultats scientifiques qui sous-tendent les orientations et les choix pédagogiques à la base de l'outil pédagogique présenté ci-dessous.

\section{Présentation de Clicfrançais}

Ancrées dans des situations concrètes du monde du travail, les activités de Clicfrançais [www.clicfrancais.com] visent, de façon concomitante, à stimuler les élèves à l'apprentissage de la langue orale et écrite ainsi qu'à leur permettre de développer des habiletés favorisant leur intégration socioprofessionnelle, particulièrement au moment de leur stage. Elles rejoignent le cadre de l'approche orientante, puisqu'elles cherchent à intégrer des notions de français à des activités reliées à l'information, à l'orientation et à la formation professionnelles.

Les projets proposés poursuivent plusieurs buts en termes de participation, de responsabilisation et d'initiative chez l'élève. Ainsi, par son caractère interactif, l'outil pédagogique permet, d'une part, le développement d'une certaine autonomie chez l'élève, en le faisant cheminer à son propre rythme, selon une approche de pédagogie différenciée. Un soutien constant de la part de l'enseignant est toutefois recommandé. D'autre part, sur le plan pédagogique, les projets visent à éveiller une prise de conscience de l'utilité, à court ou à moyen terme, des apprentissages scolaires, notamment par l'engagement cognitif de l'élève pour une consolidation de ses acquis en français, par un transfert possible de ses apprentissages en situations authentiques de travail et par une attitude positive envers le milieu de travail lors des stages. Les différentes tâches en vue de l'acquisition de connaissances sont souvent à effectuer en milieu de travail, sous forme de cueillette d'informations, de lectures ou d'entrevues par exemple.

Ne prenant pas les technologies de l'information et de la communication (TIC) pour la panacée, ces tâches peuvent, dans la recherche de solutions aux difficultés des élèves, constituer un atout pour l'apprentissage: d'une part, parce qu'elles ont un fort pouvoir d'attraction chez les jeunes (Centre francophone d'informatisation des organisations, 2004); d'autre part, parce qu'elles peuvent constituer un facilitateur tant sur le plan affectif (augmentation de la motivation) que sur le plan cognitif (ajout d'un aspect dynamique au contenu, caractère interactif, autocorrection possible, rétroaction synchrone/asynchrone, outils de soutien, recours possible aux stratégies de lecture et d'écriture en mode synchrone, et illustrations). 
Par ailleurs, pour ce qui est de la motivation, les avis sont partagés. Desmarais (1998) et Harvey (1999) ont montré que l'utilisation des TIC peut contribuer à augmenter la motivation à l'apprentissage, alors que pour Karsenti, les TIC en elles-mêmes ne favorisent pas nécessairement la motivation ou le rendement scolaire (2003, p. 30). Selon Depover (Meunier, 1997), une bonne utilisation des TIC offre des avantages sur le plan de l'apprentissage individualisé, de l'adaptation au rythme et aux stratégies d'apprentissage de l'apprenant, des rétroactions sur mesure et de l'évaluation en cours d'apprentissage; elles augmentent, selon Godard (1998), dans certains cas, la motivation à la lecture. Les TIC facilitent également les apprentissages sur le plan psychomoteur pour les élèves qui éprouvent des difficultés avec la motricité fine, la manipulation de la souris s'avérant plus simple qu'un crayon ou un stylo. Rappelons aussi que la maîtrise des compétences en lien avec les TIC constitue une exigence pour intégrer le marché du travail du $21^{\mathrm{e}}$ siècle.

\subsection{Caractéristiques et contenus de l'outil}

Selon la Société de développement de la main-d'œuvre du Québec, les employés doivent démontrer une capacité à travailler en équipe, une habileté à communiquer oralement et par écrit, un désir d'apprendre, un sens des responsabilités, une certaine créativité, un esprit d'initiative, une flexibilité et une polyvalence. Considérant cette importance de préparer les jeunes aux exigences du monde du travail, nous avons mis de l'avant des stratégies pédagogiques en vue du développement des compétences d'employabilité. Nous avons préconisé une pédagogie par projets, qui maintient un équilibre entre les activités à faire seul, celles qui mènent à un produit commun et les travaux d'équipe. Les cours magistraux y ont aussi leur place. Une mise en situation collective amorce chaque activité, pour permettre le rappel des connaissances générales et langagières et pour susciter l'intérêt. Les consignes sont traitées en grand groupe afin de soutenir l'élève qui éprouve de plus grandes difficultés de compréhension. Le véhicule du courriel et du forum de discussion offre la possibilité de poser toutes les questions nécessaires à l'enseignant, sans que l'élève ne se sente jugé.

Par le choix de ses contenus et de ses orientations Clicfrançais s'inscrit dans les visées du Programme de formation de l'école québécoise (Ministère de l'Éducation du Québec, 2001) (Annexe), mettant l'élève au centre de ses apprentissages. Il touche plus particulièrement le domaine général Orientation et entrepreneuriat et le domaine des Langues, français langue d'enseignement. La centaine d'activités proposées rejoint l'une ou l'autre des neuf compétences transversales.

Les activités supposent que les employeurs soient prêts à s'investir dans la formation de base des jeunes, tel que le préconise le Renouveau pédagogique, en engageant la communauté extra-scolaire dans la formation des jeunes.

Projets pédagogiques - Clicfrançais propose plus de 50 activités d'apprentissage en ligne (auxquelles s'ajoutent une cinquantaine d'activités sur fiches reproducti- 
bles) visant la réalisation de trois projets pédagogiques: 1) Recherche d'emploi, 2) Mini-entreprise et 3) Magazine. Ce dernier est le fruit d'une communauté de pratique, nommée Réseau québécois de l'insertion sociale et professionnelle des jeunes, issue des échanges autour des deux premiers projets. Il est né après la première année d'utilisation et ne fait pas partie de l'évaluation dont il sera question.

Le projet Recherche d'emploi (Annexe 1) met en valeur la démarche complexe et dynamique à laquelle il faut préparer les jeunes pour leur insertion socioprofessionnelle. Ainsi, sont proposés des parcours visant le développement de plusieurs compétences: aider l'élève à mieux se connaître et à mieux connaître le marché du travail et ses exigences. Dans ce but, on y trouve des activités pour découvrir des valeurs recherchées par les employeurs, demander une carte d'assurance sociale, comprendre et choisir des offres d'emploi pertinentes, remplir un formulaire de demande d'emploi, rédiger une lettre de présentation et un curriculum vitæ adapté à un poste convoité, comprendre un organigramme ou lire un chèque de paye.

Le projet Mini-entreprise (Annexe 2) s'inspire du succès des projets de création de mini-entreprises déjà existants dans certaines écoles secondaires. La participation à un tel projet peut être formatrice et motivante pour l'élève, puisque riche en expériences, en coopération et en créativité. En s'engageant dans le projet qui comprend 29 activités, l'élève est amené à participer à toutes les étapes, de l'idéation aux relations avec les clients, pour lesquelles Clicfrançais offre les outils nécessaires: donner ses idées, partager son opinion avec celles des autres, visiter une entreprise, rédiger des lettres destinées au directeur de l'école et à des commanditaires potentiels, inventer un slogan publicitaire, créer une affiche promotionnelle, gérer une caisse, interagir avec un client, etc.

Quant au projet Magazine, ses activités amènent l'élève à rédiger un texte et à contribuer à la création d'un magazine publié en ligne. Par cette voie, l'élève peut mieux se connaître et structurer son identité; exploiter l'information (se l'approprier, en tirer profit); exercer son jugement critique (construire son opinion, exprimer son jugement et relativiser); écrire des textes variés (reportage, critique, chronique, entrevue, éditorial, poème, etc.); échanger avec d'autres personnes sur les productions réalisées et se familiariser avec des stratégies d'écriture présentées sous forme de capsules d'aide en mode interactif, auxquelles il peut recourir en tout temps.

Plusieurs outils, dont il sera question ci-dessous, permettent de soutenir l'élève, faible lecteur: cyberportfolio, carnet de notes, dictionnaire, stratégies d'apprentissage et d'autorégulation sous forme de bédé ou d'animation, messagerie et forum de discussion.

\subsection{Outils d'accompagnement}

Ils se divisent en trois catégories: les interfaces, les outils d'apprentissage et les outils d'enseignement. 
Les interfaces • Trois interfaces ont été mises à la disposition des utilisateurs. La première interface, réservée à l'enseignant, inclut le dossier des élèves, la planification pédagogique (parcours d'apprentissage, guide d'accompagnement et carnet de notes); des outils de communication avec messagerie et forums et une section Aide incluant le fonctionnement du site avec possibilité d'envoyer un message au webmestre.

L'interface Élève regroupe les projets de classe; le cyberportfolio (travaux réalisés, dictionnaire personnel et carnet de notes); les outils de communication, une section Publication et Aide pour dépanner sur le fonctionnement du site, pour donner des stratégies et des conseils.

Quant à l'interface Administrateur, elle contient les demandes d'inscription; les outils de communication, la création et la gestion des comptes d'accès et un questionnaire d'évaluation (voir Annexe 2) servant d'outil de suivi.

Les outils d'apprentissage • Chaque élève possède sa page d'accueil personnalisée. Le portfolio d'apprentissage inclut les travaux terminés, un carnet de notes en ligne et un dictionnaire personnel géré par l'élève. Au cours des étapes d'un projet, l'élève qui recherche une définition peut cliquer sur des mots du vocabulaire courant ou sur ceux qui sont liés au monde du travail. Il peut ainsi réaliser son dictionnaire personnalisé en choisissant d'y inclure ces mots. Deux moyens de communication sont proposés à l'élève: une messagerie interne, qui le met en contact avec tous les élèves et les enseignants de sa classe inscrits au site, et un forum de discussion réservé à l'école, qui permet des échanges enrichissants entre les élèves et les enseignants. Afin de valoriser les efforts des jeunes, un espace géré par l'enseignant permet la publication de certains travaux. Avec l'approbation du jeune ou à sa demande, l'enseignant peut décider de rendre publics certains travaux qui servent d'exemples aux autres, tant par la qualité du contenu ou de la présentation que par l'effort fourni par l'élève.

Enfin, dans la section Aide, l'élève trouvera du soutien sur le fonctionnement du site. Dans une banque, il pourra également puiser des stratégies, par exemple en lecture et écriture, et des conseils qui lui seront utiles au cours de la réalisation des projets.

Les outils d'enseignement • Finalement, des outils de soutien sont proposés aux enseignants pour faciliter la gestion des dossiers des élèves et le suivi de leur cheminement dans le site. Une section facilitant sa planification pédagogique inclut le guide d'accompagnement, le carnet de notes et l'accès aux activités d'apprentissage comme elles sont présentées à l'élève. Quant aux espaces de communication, un forum réunit tous les enseignants inscrits au site, un autre est réservé à l'école où l'enseignant peut animer des discussions avec ses élèves ainsi qu'une messagerie privée. Des outils d'assistance, tels que le fonctionnement du site, le soutien pédagogique et le support technique offert par le webmestre sont prévus. 
Avec ce tour d'horizon des différentes composantes de l'outil pédagogique en ligne, nous espérons faire ressortir que Clicfrançais a été conçu non seulement pour répondre aux besoins des élèves sur le plan du soutien à l'apprentissage, mais aussi comme aide aux enseignants, novices avec les TIC dans certains cas, ou à court de matériel.

Comme les réalisations sont soumises à une évaluation périodique par le Centre de transfert pour la réussite éducative du Québec, nous rapportons dans la prochaine section les résultats d'une évaluation de l'outil pédagogique, effectuée au cours des premiers mois de sa mise en ligne.

\section{Résultats}

L'évaluation de Clicfrançais réalisée auprès de ses utilisateurs, élèves et enseignants, vise à mesurer l'efficacité de l'outil, le niveau de satisfaction des utilisateurs et son impact. Deux questionnaires d'évaluation (version enseignant/élève) ont été utilisés pour la collecte des données.

Rappelons que les projets du Centre de transfert pour la réussite éducative du Québec visent à favoriser la réussite éducative et à lutter contre l'échec et l'abandon scolaires. Pour ce faire, les réalisations financées doivent s'inscrire dans les objectifs visés, être adaptées aux clientèles ciblées, servir effectivement à celles-ci, répondre aux besoins des utilisateurs et avoir un impact réel sur eux. Quant à ses outils, le Centre de transfert pour la réussite éducative du Québec s'est doté d'un cadre d'évaluation guidé et orienté par huit critères, regroupés en trois catégories (Landry, 2005, p. 10) (Annexe) dont les questionnaires sont inspirés. Ceux-ci restent disponibles en tout temps sur le site.

Le questionnaire d'évaluation destiné aux enseignants comporte 21 énoncés répartis dans les catégories: 1) atteinte des objectifs, 2) niveau de satisfaction et 3) impact de l'outil. Une échelle de réponse de type Likert à six gradations allant de Totalement en accord à Totalement en désaccord est utilisée. Sept enseignants de sept écoles secondaires y ont répondu. Pour la version élève, 21 énoncés sont également présentés avec une échelle de Likert à quatre gradations allant de Totalement d'accord à Totalement en désaccord. Vingt-six élèves (17 garçons et neuf filles) de neuf écoles secondaires y ont participé.

Dans les deux cas, seuls les 17 premiers énoncés ont été analysés, les quatre autres concernant l'utilisation des TIC et non le contenu de Clicfrançais. 


\subsection{Efficacité}

Tableau 1

Atteinte des objectifs selon les enseignants $(n=7)$

\begin{tabular}{|c|c|c|c|c|c|c|}
\hline & $\begin{array}{c}\text { Totalement } \\
\text { d'accord }\end{array}$ & $\begin{array}{c}\text { Assez } \\
\text { d'accord }\end{array}$ & $\begin{array}{l}\text { Un peu } \\
\text { d'accord }\end{array}$ & $\begin{array}{l}\text { Un peu en } \\
\text { désaccord }\end{array}$ & $\begin{array}{l}\text { Assez en } \\
\text { désaccord }\end{array}$ & $\begin{array}{c}\text { Totalement } \\
\text { en désaccord }\end{array}$ \\
\hline $\begin{array}{l}\text { 1. Le site répond à un besoin } \\
\text { en matériel pédagogique } \\
\text { adapté aux } \\
\text { caractéristiques des élèves }\end{array}$ & 4 & 3 & 0 & 0 & 0 & 0 \\
\hline $\begin{array}{l}\text { 2. Le site et son contenu } \\
\text { pédagogique sont } \\
\text { attrayants, interactifs et } \\
\text { dynamiques }\end{array}$ & 3 & 4 & 0 & 0 & 0 & 0 \\
\hline $\begin{array}{l}\text { 3. Le site contribue à motiver } \\
\text { les élèves à l'apprentissage } \\
\text { du français }\end{array}$ & 2 & 1 & 4 & 0 & 0 & 0 \\
\hline $\begin{array}{l}\text { 4. Le site développe des } \\
\text { habiletés de préparation et } \\
\text { d'intégration au marché du } \\
\text { travail chez les élèves }\end{array}$ & 2 & 4 & 1 & 0 & 0 & 0 \\
\hline
\end{tabular}

L'évaluation de l'efficacité permet de vérifier dans quelle mesure le produit a atteint ses objectifs. Une évaluation du produit permettra de juger de son contenu, de sa forme, de sa qualité et de sa diffusion, entre autres par la mesure de la satisfaction de la clientèle (Landry, 2005, p. 9).

\subsubsection{Atteinte des objectifs}

Les réponses indiquent que les enseignants sont totalement d'accord (4) ou assez d'accord (3) avec l'énoncé 1: Le site répond à un besoin en matériel pédagogique adapté aux caractéristiques des élèves. Questionnés sur le dynamisme, l'interactivité et l'attrait du site et de son contenu pédagogique (énoncé 2), les enseignants se disent totalement d'accord (3) ou assez d'accord (4). À l'énoncé 4, Le site développe des habiletés de préparation et d'intégration au marché du travail chez les élèves, six enseignants sur sept sont totalement d'accord ou assez d'accord, un seul étant un peu d'accord. Nous croyons avoir là un appui à une de nos visées premières, l'intégration des compétences de préparation au monde du travail dans les matières de base. Enfin, à des degrés divers, les enseignants sont d'accord pour dire que le site contribue à motiver les élèves à l'apprentissage du français (énoncé 3 ) : deux enseignants sont totalement d'accord, un est assez d'accord et quatre sont un peu d'accord. Toutefois, dans les commentaires transmis par les enseignants qui se sont prononcés comme un peu d'accord, on lit: Jusqu'à maintenant, les commentaires des élèves sont très positifs [...] Un bon degré de compréhension en lecture est nécessaire pour une bonne motivation. Certains élèves ayant de plus grandes difficultés se découragent plus rapidement. Ces commentaires nous font penser que le degré de 
Tableau 2

Atteinte des objectifs selon les élèves $(n=26)$

\begin{tabular}{lcccc}
\hline & $\begin{array}{c}\text { Totalement } \\
\text { d'accord }\end{array}$ & $\begin{array}{c}\text { Assez } \\
\text { d'accord }\end{array}$ & $\begin{array}{c}\text { Assez en } \\
\text { désaccord }\end{array}$ & $\begin{array}{c}\text { Totalement } \\
\text { en désaccord }\end{array}$ \\
\hline $\begin{array}{l}\text { 1. Les activités proposées dans Clicfrançais me } \\
\text { plaisent, elles sont dynamiques et }\end{array}$ & 9 & 15 & 1 & 1 \\
interactives & 21 & 4 & 1 & 0 \\
$\begin{array}{l}\text { 2. J'aime réaliser des projets à l'ordinateur. } \\
\text { 3. Je me sens plus motivé(e) à apprendre } \\
\text { quand j'utilise Clicfrançais }\end{array}$ & 13 & 7 & 6 & 0 \\
$\begin{array}{l}\text { 4. Les activités présentées dans Clicfrançais } \\
\text { me permettent d'être mieux préparé(e) pour } \\
\text { mon futur emploi }\end{array}$ & 13 & 11 & 1 & 1 \\
\hline
\end{tabular}

difficulté reliée à la lisibilité de certaines consignes ou de certains textes fait obstacle à la motivation.

Quant aux élèves, à l'énoncé 1 Les activités proposées dans Clicfrançais me plaisent, elles sont dynamiques et interactives, 9/26 (30\%) se disent totalement d'accord ou 15/26 (55\%), assez d'accord; deux d'entre eux ne semblent pas d'accord avec l'énoncé. À l'énoncé 4 visant à vérifier la prise de conscience de l'utilité des apprentissages scolaires pour mieux se préparer à leur futur emploi, réalisés dans Clicfrançais, 13/26 (50\%) sont totalement d'accord ou 11/26 (40\%), assez d'accord. Deux n'y ont toutefois pas trouvé d'intérêt. Sachant que les jeunes de cet âge, particulièrement la population de ces programmes, ont de la difficulté à se dessiner un projet d'avenir, il se pourrait que le choix d'un futur emploi ne soit pas encore arrêté. Les activités de découverte (mieux se connaître, explorer des métiers, etc.) proposées dans Clicfrançais cherchent précisément à aider les élèves dans cette démarche. Lorsqu'il est question d'aimer réaliser des projets à l'ordinateur (énoncé 2), 21/26 (77\%) se prononcent totalement d'accord ou 4/26 (11\%), assez d'accord. Enfin, la moitié (13/26) se disent totalement d'accord et 7/26 (26\%), assez d'accord avec l'énoncé 3 : Je me sens plus motivé(e) à apprendre quand j'utilise Clicfrançais. Par contre, six élèves ne semblent pas plus motivés. Cette réponse rejoint celle des enseignants sur le même objet. Plusieurs explications peuvent être évoquées, soit le degré de difficulté trop élevé des tâches demandées, soit le sentiment de faible compétence ou le manque d'intérêt, voire l'absence d'engagement, de l'élève dans son apprentissage.

\subsubsection{Niveau de satisfaction}

Six enseignants sur sept sont totalement d'accord ou assez d'accord pour affirmer que le contenu du site est bien adapté aux élèves (énoncé 5). Les enseignants se déclarent totalement d'accord (1) ou assez d'accord (6) sur le fait que les contenus d'apprentissage proposés sont pertinents et de qualité et que le guide d'accompagnement appuie adéquatement (2/7, totalement d'accord; 5/7, assez d'accord) les activités du site et la démarche pédagogique proposée (énoncés 6 et 7). 
Debeurme, G. et Landry, l. 79

Tableau 3

Niveau de satisfaction selon les enseignants $(n=7)$

\begin{tabular}{|c|c|c|c|c|c|c|}
\hline - & $\begin{array}{l}\text { Totalement } \\
\text { d'accord }\end{array}$ & $\begin{array}{c}\text { Assez } \\
\text { d'accord }\end{array}$ & $\begin{array}{l}\text { Un peu } \\
\text { d'accord }\end{array}$ & $\begin{array}{l}\text { Un peu en } \\
\text { désaccord }\end{array}$ & $\begin{array}{l}\text { Assez en } \\
\text { désaccord }\end{array}$ & $\begin{array}{l}\text { Totalement } \\
\text { en désaccord }\end{array}$ \\
\hline $\begin{array}{l}\text { 5. Le contenu est bien } \\
\text { adapté aux élèves }\end{array}$ & 2 & 4 & 1 & 0 & 0 & 0 \\
\hline $\begin{array}{l}\text { 6. Les contenus } \\
\text { d'apprentissage } \\
\text { proposés sont pertinents } \\
\text { et de qualité }\end{array}$ & 1 & 6 & 0 & 0 & 0 & 0 \\
\hline $\begin{array}{l}\text { 7. Le guide } \\
\text { d'accompagnement } \\
\text { appuie adéquatement } \\
\text { les activités du site et la } \\
\text { démarche pédagogique } \\
\text { proposée }\end{array}$ & 2 & 5 & 0 & 0 & 0 & 0 \\
\hline $\begin{array}{l}\text { 8. Les projets s'insèrent } \\
\text { bien dans le contexte de } \\
\text { la classe, dans le } \\
\text { respect des objectifs du } \\
\text { MÉLS et du temps } \\
\text { accordé pour atteindre } \\
\text { les compétences visées }\end{array}$ & 2 & 2 & 3 & 0 & 0 & 0 \\
\hline $\begin{array}{l}\text { 9. Il est simple d'utiliser les } \\
\text { multiples fonctionnalités } \\
\text { du site (messagerie, } \\
\text { forums, carnet de notes, } \\
\text { révision et publication } \\
\text { des travaux, etc.) }\end{array}$ & 2 & 2 & 2 & 1 & 0 & 0 \\
\hline $\begin{array}{l}\text { 10. Il est facile de naviguer } \\
\text { dans le site }\end{array}$ & 1 & 6 & 0 & 0 & 0 & 0 \\
\hline $\begin{array}{l}\text { 11. Le concept visuel du site } \\
\text { est attrayant, agréable } \\
\text { et harmonieux }\end{array}$ & 4 & 3 & 0 & 0 & 0 & 0 \\
\hline $\begin{array}{l}\text { 12. Dans votre école, vous } \\
\text { disposez de } \\
\text { l'équipement } \\
\text { informatique et de la } \\
\text { configuration requis } \\
\text { pour profiter pleinement } \\
\text { du site }\end{array}$ & 5 & 1 & 0 & 0 & 1 & 0 \\
\hline $\begin{array}{l}\text { 13. De façon générale, vous } \\
\text { êtes satisfait du site } \\
\text { (intérêt, utilité, } \\
\text { originalité) }\end{array}$ & 2 & 4 & 1 & 0 & 0 & 0 \\
\hline
\end{tabular}

Par contre, en ce qui concerne l'énoncé 8 , même si quatre enseignants sont totalement d'accord (2) ou assez d'accord (2) avec l'énoncé, trois des sept enseignants se disent un peu d'accord avec le fait que les projets s'insèrent bien dans le contexte 
de la classe, dans le respect des objectifs du ministère de l'Éducation, du Loisir et du Sport, et du temps accordé pour atteindre les compétences visées. Le temps d'adaptation qu'exige tout nouvel outil ainsi qu'une familiarisation avec son contenu et son utilisation pour une meilleure intégration dans les projets de classe habituels ne seraient-ils pas une explication plausible à la réponse mitigée de ces derniers?

Les enseignants se disent satisfaits de l'ergonomie et de la convivialité du site. À l'énoncé 10, Il est facile de naviguer dans le site, un enseignant indique qu'il est totalement d'accord; les six autres sont assez d'accord. De plus, lorsqu'on leur demande de se prononcer sur la simplicité d'utilisation des multiples fonctionnalités du site (messagerie, forums, carnet de notes, révision et publication des travaux, etc.) (énoncé 9), deux enseignants se déclarent totalement d'accord; deux, assez d'accord; deux, un peu d'accord et un, un peu en désaccord. Ces réponses dépendent, à notre avis, en partie, du degré de familiarité des utilisateurs avec les technologies.

La qualité visuelle du site (attrayant, agréable et harmonieux) est appréciée par les sept enseignants (énoncé 11): quatre sont totalement d'accord et trois assez d'accord.

Dans son ensemble, le site est apprécié par les enseignants (énoncé 13): six enseignants sur sept affirment être satisfaits totalement (2), ou assez (4) de l'intérêt, de l'utilité et de l'originalité du site; un seul ne semble pas de cet avis, mais nous n'avons recueilli aucun commentaire. Ils illustrent leur appréciation générale du site par un pourcentage allant de $75 \%$ à $90 \%$, avec une moyenne générale de $81 \%$.

Quant à l'énoncé 12, il n'est pas commenté parce qu'il ne concerne pas spécifiquement l'outil pédagogique.

\section{Tableau 4}

Niveau de satisfaction selon les élèves $(n=26)$

\begin{tabular}{|c|c|c|c|c|}
\hline & $\begin{array}{l}\text { Totalement } \\
\text { d'accord }\end{array}$ & $\begin{array}{l}\text { Assez } \\
\text { d'accord }\end{array}$ & $\begin{array}{l}\text { Assez en } \\
\text { désaccord }\end{array}$ & $\begin{array}{l}\text { Totalement } \\
\text { en désaccord }\end{array}$ \\
\hline $\begin{array}{l}\text { 5. La recherche d'emploi et la création d'une } \\
\text { entreprise sont des sujets qui m'intéressent }\end{array}$ & 17 & 9 & 0 & 0 \\
\hline $\begin{array}{l}\text { 6. Je crois que les projets Recherche d'emploi et } \\
\text { Mini-entreprise sont utiles }\end{array}$ & 11 & 12 & 1 & 2 \\
\hline $\begin{array}{l}\text { 7. J'ai trouvé les étapes du projet faciles à } \\
\text { réaliser }\end{array}$ & 13 & 11 & 2 & 0 \\
\hline 8. J'aime les couleurs du site et les illustrations. & 12 & 12 & 1 & 0 \\
\hline $\begin{array}{l}\text { 9. Le site et ses outils (carnet de notes, } \\
\text { messagerie, forums, dictionnaire personnel, } \\
\text { etc.) sont simples à utiliser }\end{array}$ & 21 & 4 & 0 & 1 \\
\hline $\begin{array}{l}\text { 10. Les textes sont faciles à lire et je les } \\
\text { comprends bien }\end{array}$ & 18 & 8 & 0 & 0 \\
\hline 11. Il est facile de naviguer dans Clicfrançais & 18 & 5 & 2 & 1 \\
\hline 12. De façon générale, j'aime Clicfrançais & 10 & 15 & 1 & 0 \\
\hline
\end{tabular}


Dix-sept des 26 sujets sont totalement d'accord et neuf (34,6\%), assez d'accord avec l'énoncé 5 : le choix des projets semble donc adéquat et correspondre à leurs intérêts. Ces résultats sont appuyés par les réponses à l'énoncé 6, puisque 11 des 26 élèves se disent totalement d'accord et 12, assez d'accord, pour croire que les deux projets sont utiles. Rappelons que, pour Viau (1994), la perception positive de la valeur d'une tâche peut contribuer à la motivation à apprendre. Par contre, il ne faut pas négliger les trois répondants qui ne s'intéressent pas ou peu aux deux projets. S'agit-il d'un manque d'intérêt général ou plutôt spécifique aux deux thèmes suggérés? Dans une prochaine évaluation, il nous faudra donc ajouter une question pour connaître les raisons de ce manque d'intérêt.

Les élèves partagent l'avis des enseignants sur l'ergonomie et la convivialité du site (énoncé 9) : 21 sont totalement d'accord et quatre, assez d'accord. Aussi, ont-ils de la facilité à utiliser les fonctionnalités du site (messagerie, forum, dictionnaire personnel, etc.) (énoncé 11). Pas étonnant, si l'on se fie à une enquête menée par le Centre francophone d'informatisation des organisations (2004), qui rapporte que $88 \%$ des adolescents de 12 à 17 ans sont des fervents du Net et qu'ils y consacrent environ huit heures/semaine. Pour leur part, 18 élèves sont totalement d'accord et huit, assez d'accord, avec l'énoncé 10: Les textes sont faciles à lire et je les comprends bien.

Chez les élèves, 13 se disent totalement d'accord et 11 (42\%), assez d'accord avec l'énoncé 7 : J'ai trouvé les étapes du projet faciles à réaliser. Toutefois, deux d'entre eux ne sont pas de cet avis: la réalisation ne leur semble donc pas si évidente. $\mathrm{Ne}$ s'agirait-il pas de mettre ces réponses en relation avec l'énoncé précédent?

Douze élèves sont totalement d'accord et 12, assez d'accord, pour apprécier les couleurs du site et les illustrations. Rappelons que les couleurs du site et le concept des personnages (illustrations) (énoncé 8) ont été choisis par une classe d'élèves en insertion sociale et professionnelle des jeunes.

Enfin, 25 des 26 élèves sont totalement d'accord (10) ou assez d'accord (15) pour dire que, de façon générale (énoncé 12), ils aiment Clicfrançais.

\subsection{Impact de l'outil}

L'impact se mesure par son effet sur la clientèle visée (Landry, 2005, p. 9). Il peut être de deux ordres: direct ou hors cibles. Les réponses des enseignants et des élèves ne concernent pas les effets hors cibles.

À l'énoncé 14, Le site est un apport significatif à l'amélioration ou au renouvellement des pratiques d'enseignement en ISPJ, les avis sont favorables, avec quatre enseignants totalement d'accord et trois assez d'accord. À l'énoncé 15, L'utilisation de cet outil pour l'enseignement du français a fait hausser le taux de motivation pour son apprentissage chez vos élèves, un enseignant s'est déclaré totalement d'accord; trois enseignants, assez d'accord et trois enseignants, un peu d'accord. Cette dernière donnée confirme que l'intégration des TIC ne suffit pas à augmenter la motivation (Karsenti, 2003: voir Pellerin, 2005, p. 70; Viau, 1999) et que d'autres facteurs 
82 Revue des sciences de l'éducation, volume 35, n 1, 2009

Tableau 5

Impacts de l'outil selon les enseignants $(n=7)$

\begin{tabular}{|c|c|c|c|c|c|c|}
\hline & $\begin{array}{l}\text { Totalement } \\
\text { d'accord }\end{array}$ & $\begin{array}{c}\text { Assez } \\
\text { d'accord }\end{array}$ & $\begin{array}{l}\text { Un peu } \\
\text { d'accord }\end{array}$ & $\begin{array}{l}\text { Un peu en } \\
\text { désaccord }\end{array}$ & $\begin{array}{l}\text { Assez en } \\
\text { désaccord }\end{array}$ & $\begin{array}{c}\text { Totalement } \\
\text { en désaccord }\end{array}$ \\
\hline $\begin{array}{l}\text { 14. Le site est un apport } \\
\text { significatif à } \\
\text { l'amélioration ou au } \\
\text { renouvellement des } \\
\text { pratiques } \\
\text { d'enseignement en } \\
\text { ISPJ }\end{array}$ & 4 & 3 & 0 & 0 & 0 & 0 \\
\hline $\begin{array}{l}\text { 15. L'utilisation de cet outil } \\
\text { pour l'enseignement } \\
\text { du français a fait } \\
\text { hausser le taux de } \\
\text { motivation pour son } \\
\text { apprentissage chez vos } \\
\text { élèves }\end{array}$ & 1 & 3 & 3 & 0 & 0 & 0 \\
\hline $\begin{array}{l}\text { 16. Les projets proposés } \\
\text { dans le site ont suscité } \\
\text { beaucoup d'intérêt } \\
\text { chez vos élèves. }\end{array}$ & 2 & 4 & 0 & 1 & 0 & 0 \\
\hline
\end{tabular}

personnels liés aux perceptions de soi et de la compétence, de la valeur de la tâche et aux échecs antérieurs peuvent influencer. Par contre, les points de vue de six enseignants sont positifs (deux, totalement d'accord; quatre, assez d'accord) quant à l'intérêt suscité chez les élèves.

Tableau 6

Impacts de l'outil selon les élèves $(n=26)$

\begin{tabular}{lcccc}
\hline & $\begin{array}{c}\text { Totalement } \\
\text { d'accord }\end{array}$ & $\begin{array}{c}\text { Assez } \\
\text { d'accord }\end{array}$ & $\begin{array}{c}\text { Assez en } \\
\text { désaccord }\end{array}$ & $\begin{array}{c}\text { Totalement } \\
\text { en désaccord }\end{array}$ \\
\hline $\begin{array}{l}\text { 13. Depuis que je participe à Clicfrançais, } \\
\text { j'ai amélioré mes connaissances et mes } \\
\text { habiletés en français }\end{array}$ & 13 & 10 & 0 & 1 \\
$\begin{array}{l}\text { 14. Depuis que je participe à Clicfrançais, } \\
\text { j'ai augmenté mes connaissances sur } \\
\text { le monde du travail (ses exigences, ses } \\
\text { possibilités, etc.) }\end{array}$ & 11 & 12 & 1 & 2 \\
$\begin{array}{l}\text { 15. Depuis que je participe à Clicfrançais, } \\
\text { je me connais mieux (mes intérêts, } \\
\text { mes compétences, mes habiletés } \\
\text { d'employabilité, etc.) }\end{array}$ & 8 & 13 & 3 & 2 \\
$\begin{array}{l}\text { 16. J'ai le goût de faire des activités en } \\
\text { français dans Clicfrançais }\end{array}$ & 10 & 13 & 3 & 0 \\
$\begin{array}{l}\text { 17. Je vais souvent au local d'informatique } \\
\text { de mon école }\end{array}$ & 8 & 10 & 5 & 2 \\
\hline
\end{tabular}


Parmi les 26 élèves, la moitié se sont prononcés comme totalement d'accord et 10 (37\%), assez d'accord avec l'énoncé 13: Depuis que je participe à Clicfrançais, j'ai amélioré mes connaissances et mes habiletés en français. Même si nous ne pouvons localiser cette amélioration, nous croyons qu'une telle prise de conscience peut susciter une perception positive et favoriser l'apprentissage. À l'énoncé 14, Depuis que je participe à Clicfrançais, j'ai augmenté mes connaissances sur le monde du travail (ses exigences, ses possibilités...), 11 sujets se disent totalement d'accord et 12, assez d'accord, contre deux, totalement en désaccord, et qui n'en auraient donc pas tiré profit. Rappelons que ces deux énoncés concernent les deux cibles prioritaires à l'origine de l'élaboration de l'outil didactique, qui résultent des données de recherche.

Quant à l'énoncé 15, Depuis que je participe à Clicfrançais, je me connais mieux (mes intérêts, mes compétences, mes habiletés d'employabilité...), huit répondants se disent totalement d'accord et 13, assez d'accord. Par contre, cinq d'entre eux ne semblent pas avoir trouvé satisfaction par rapport à cet aspect.

À l'énoncé 16, J'ai le goût de faire des activités en français dans Clicfrançais, 10 sont totalement d'accord et 13, assez d'accord. Quatre sujets ne semblent pas trouver le goût de faire les activités (assez en désaccord). Ces réponses terminent l'analyse des données.

\section{Discussion}

En ce qui concerne l'atteinte des objectifs, enseignants et élèves ont généralement répondu favorablement. Il appert que cet outil didactique en ligne répond à un besoin, problème traité au début de l'article. Le contenu semble correspondre aux intérêts des jeunes auxquels il est destiné. Lors d'une première pré-expérimentation en 2003 sur les contenus pédagogiques du projet Recherche d'emploi, les commentaires de cinq enseignants en Insertion sociale et professionnelle des jeunes faisaient déjà état de la pertinence des projets et de leur intérêt à utiliser ce produit. À titre complémentaire, ces opinions sont appuyées par des commentaires d'autres enseignants qui ont visité et utilisé le site durant la même période sans avoir rempli le questionnaire:

- J'ai eu l'occasion de parcourir l'ensemble pédagogique Clicfrançais, à l'intention des étudiants du secondaire, notamment ceux inscrits en ISPJ. Je suis épatée, non seulement par la pertinence des scénarios et activités, mais également par leur richesse et leur rigueur. (H. B., orthopédagogue)

- Enfin, un nouvel outil pour soutenir l'intérêt des jeunes! (J. S., enseignante en ISPJ)

- Merci pour ce site qui a capté l'intérêt des élèves en début d'année et qui montre le sérieux du marché du travail [...] Au plaisir de continuer l'année 2005-2006! (P. L., enseignant en ISPJ)

Par contre, en ce qui concerne la motivation pour apprendre le français, la partie n'est pas gagnée, même si les commentaires de la plupart des élèves sont favorables. 
En effet, $23 \%$ des élèves (tous des garçons) ne le sont pas davantage. Rappelons que nous avons effectué l'évaluation avant qu'un projet complet ne soit réalisé. Toutefois, nous sommes conscientes que l'effet peut jouer dans l'autre sens: la nouveauté de l'outil, en ligne par surcroît, peut avoir suscité un enthousiasme qui risque de se perdre à l'usage.

Quant au degré de satisfaction, l'ensemble des réponses reliées au contenu et à la forme est positif. Comme nous l'avons rapporté plus tôt, le choix des contenus et celui de leur organisation ont été guidés par nos recherches antérieures, par une évaluation de la part des enseignants ainsi que par des critères pédagogiques s'inscrivant dans les orientations ministérielles. Lors de la modélisation des activités, dans la mesure du possible, nous avons tenté de respecter les caractéristiques citées par Viau (1999, p. 19) pour créer des activités motivantes (signifiantes, diversifiées, intégrées dans un projet global, représentant un défi, responsabilisant l'élève, exigeantes sur le plan cognitif, collaboratives, et dotées de consignes claires). Enfin, la qualité visuelle a été assurée par une firme informatique spécialisée.

Quant à l'impact, les avis des élèves et des enseignants convergent pour ce qui est de l'amélioration des habiletés de préparation au travail. Nous faisons l'hypothèse qu'il reste à tisser un lien entre les observations des enseignants sur l'intérêt chez les élèves et ces derniers qui se disent assez d'accord que leurs connaissances en français et sur le monde du travail se sont améliorées. N'aurait-on pas là atteint un des objectifs premiers du programme d'insertion socioprofessionnelle?

\section{Conclusion}

À la suite d'un travail intensif en partenariat, soutenu par une équipe dynamique et engagée pour la réussite des jeunes à risque d'échec scolaire et social, nous osons croire que l'outil pédagogique Clicfrançais contribue, à sa manière et modestement, à la réussite de certains élèves, de par la réponse qu'il a pu offrir jusque-là à certains besoins concernant les connaissances du monde du travail et les apprentissages de base, ainsi qu'à ceux de leurs enseignants en matière de support pédagogique. Issue d'un transfert de résultats de recherches, cette réalisation constitue un exemple de rapprochement possible entre le monde universitaire et le milieu scolaire, auquel les élèves ont collaboré grâce à la validation de certaines activités, à leur participation au choix des couleurs, des personnages et du nom du site.

Selon les statistiques relevées entre avril 2004 et novembre 2006, 1061 enseignants et 2502 élèves ont été inscrits sur le site. Certains d'entre eux l'exploitent intégralement, d'autres partiellement. D'ailleurs, le nombre d'inscrits dépasse largement les objectifs fixés par le Centre de transfert pour la réussite éducative du Québec, soit de rejoindre $10 \%$ de la clientèle cible. Lors de sa conception, Clicfrançais visait les élèves âgés de 16 à 18 ans inscrits aux divers programmes adaptés (Insertion sociale et professionnelle des jeunes, Centres de formation en entreprise et récupération, Alternance études-travail). Or, depuis sa mise en ligne, la clientèle s'est élargie. En effet, Clicfrançais rejoint également les jeunes et les 
adultes en insertion socioprofessionnelle des Carrefours Jeunesse-emploi (CJE), des centres d'éducation des adultes (CEA) et des centres d'alphabétisation et d'insertion. Au Québec, de plus en plus d'élèves de 15 ans et moins sont inscrits au site; en Ontario, des enseignants assurant les cours de pré-emploi dispensés aux allophones ou à des décrocheurs potentiels utilisent le site; au NouveauBrunswick, ce sont les personnes chargées d'intégrer l'approche orientante qui exploitent le site avec leurs élèves des classes ordinaires.

Rappelons également que l'exploitation de Clicfrançais a eu un impact hors cible par la mise sur pied d'une communauté de pratique, le Réseau québécois de l'insertion sociale et professionnelle des jeunes, dont les échanges sont nombreux (partage d'outils, inventaire de ressources, et bulletin d'informations).

Par contre, malgré l'accroissement constant du nombre d'utilisateurs depuis le lancement du site, malgré les commentaires favorables, lors d'ateliers et de présentations, ainsi que la reconnaissance engendrée par deux mérites de $2^{\mathrm{e}}$ prix lors de concours, tant pour la qualité du contenu pédagogique que celle de l'outil technologique, il faut signaler des limites à cet outil.

La première concerne le nombre restreint d'utilisateurs qui constituent notre échantillon pour l'analyse ici rapportée et le moment auquel a été rempli le questionnaire, soit vers la fin d'un premier projet. La deuxième, dont nous avons à nous préoccuper sur le plan pédagogique, concerne le degré de difficulté soulevé quant à la lisibilité des textes ou des consignes, pour des élèves qui ont des troubles d'apprentissage. À cet égard, il faut préciser que le seuil de difficulté des textes a été fixé en considérant les capacités moyennes des élèves ciblés. Il faudra sans doute penser à différencier davantage certaines activités, à suggérer à l'enseignant d'adapter le matériel ou à offrir à l'élève des consignes et des textes en ligne sur support audio. Cependant, les nombreux outils d'aide intégrés (capsules d'aide sur les stratégies) peuvent soutenir le mauvais lecteur dans ses démarches. L'aide des pairs est aussi valorisée dans plusieurs activités pour faire progresser l'élève.

Finalement, et nous ne voulons pas considérer cela comme une limite mais davantage comme un défi, il reste à assurer une pérennité à Clicfrançais, où le dynamisme du site (ajouts de contenus, bonifications, nouveautés, support technique et pédagogique régulier, suivi et gestion, etc.) est synonyme d'intérêt, d'efficacité et de continuité. Pour garder le site en circulation, il faudra également prévoir des activités d'accompagnement dans le milieu.

Pour conclure, revenons sur l'expérience de transfert de connaissances. Celle-ci représente des contraintes, dont celles soulevées par Potvin (2005): le cheminement de négociation pour délimiter le terrain de compétences et les rôles de chaque partie est assez long. Aussi, tout stimulant qu'il soit, le projet s'avère très coûteux en temps pour le chercheur qui n'a que peu de reconnaissance pour ce type de contribution de la part de l'institution universitaire. Tout chercheur universitaire doit savoir que le transfert est le passage du leadership dans un projet de recherche, au partage et au partenariat. Il faut accepter que la connaissance expériencielle doit 
être partagée avec les connaissances scientifiques et se rapprocher des besoins du milieu (Potvin, 2005).

Nous sommes d'avis que Clicfrançais, issu des recherches, apporte une contribution à l'insertion des jeunes dans la société en leur offrant des outils d'aide à l'apprentissage de base de la langue, passeport pour leur réussite.

ENGLISH TITLE - An study of transfer of research results : evaluation of Clicfrançais, on-line teaching scenarios intended for socio-professional insertion

SUMMARY - Students presenting difficulties in learning language are at the centre of our preoccupations. It is paramount that intervention places these students in motivating learning contexts, by offering activities adapted to their needs and capacities. It is with this objective that Clicfrançais was elaborated within the frame of a transfer of knowledge theory, financed by the CTREQ. In this article, we present the evolution between research results and their impact on a didactic on-line tool intended for youth experiencing socio-professional insertion. We describe the contents of the tool and the results of its partial evaluation by a restricted number students and teachers.

KEY WORDS - socio-professional insertion, language learning difficulties, secondary level teaching, on-line tool, transfer of knowledge.

TítULO EN ESPAÑOL - Una experiencia de transferencia de resultados de investigación: evaluación de Clicfrançais, situaciones pedagógicas en línea destinadas a la inserción socioprofesional

RESUMEN - Desde hace varios años, los alumnos que presentan dificultades de adquisición del idioma forman parte de nuestras preocupaciones. Para ayudarlos, es primordial asegurarse que se encuentren en contextos de aprendizaje motivadores y proporcionarles actividades adaptadas a sus necesidades, incluso a sus capacidades. Es con este propósito que Clicfrançais ha sido elaborado en el marco de una transferencia de conocimientos financiada por el Centre de transfert pour la réussite éducative du Québec (CTREQ). En el presente artículo, presentamos el camino realizado a partir de los resultados de investigación hasta su culminación en una herramienta didáctica en línea, puesta a la disposición de jóvenes en inserción socioprofesional. Describimos el contenido de la herramienta y presentamos los resultados de su evaluación parcial realizada por un número limitado de alumnos y de docentes.

PALABRAS CLAVES • inserción socioprofesional, dificultades de lenguaje, enseñanza secundaria, herramienta en línea, transferencia de conocimientos.

\section{Références}

Baby, A. (2005). Pédagogie des poqués. Québec, Québec: Presses de l’Université du Québec.

Bourdon, S. et Roy, S. (2004). J'embarque quand ça me ressemble. Cadre andragogique. Québec, Québec: Gouvernement du Québec.

Centre francophone d'informatisation des organisations (CEFRIO) (2004). NetAdos 2004. Portrait des 12-17 ans sur Internet: sondage réalisé auprès des ados québécois et de leurs parents. [En ligne]. Disponible le 19 février 2008: www.cefrio.qc.ca/rapports/ NetAdos_2004_rapport.pdf 
Comité sur l'approche orientante en Estrie (2002). Pour une approche orientante intégrée. Cadre de référence. Sherbrooke, Québec: Université de Sherbrooke.

Debeurme, G. (1995). Effets d'activités d'éducation à la carrière sur les acquisitions lexicales et sur l'intérêt pour le français. Thèse de doctorat inédite, Université de Montréal, Montréal.

Debeurme, G. (1999). L'éducation à la carrière: une avenue pour le développement concomitant des habiletés langagières et de préparation au travail dans les cours de français au secondaire. Cahiers de la recherche en éducation, 6(1), 57-80.

Desmarais, L. (1998). Les technologies et l'enseignement des langues. Montréal, Québec: Logiques.

Godard, L. (1998). La littérature de jeunesse et le traitement de texte: des outils didactiques d'avenir avec Internet. Dans C. Préfontaine, L. Godard et G. Fortier (Dir.) : Pour mieux comprendre la lecture et l'écriture. Montréal, Québec: Logiques.

Harvey, D. (1999). Les Nouvelles Technologies de l'Information et des Communications (NTIC) et la formation universitaire. Les technologies de l'information et de la communication et leur avenir en éducation, 27(2).

Hoyt, K. B. (1987). Career education in transition: trends and implications for the future. Columbus, Ohio: The National Center for Research in Vocational Education.

Karsenti, T. (2003). Favoriser la motivation et la réussite en contexte scolaire : les TIC ferontelles mouche? Vie pédagogique, 127, 27-31.

Landry, I. (2005). Rapport d'évaluation 2002-2005. Scénarios d'apprentissage pour les élèves d'ISPJ, ISPMT et AET. Loretteville, Québec: Centre de transfert pour la réussite éducative du Québec (CTREQ).

Meunier, C. (1997). Points de vue sur le multimédia interactif en éducation: entretiens avec 13 spécialistes européens et nord-américains. Montréal, Québec: Éditions de la Chenelière.

Ministère de l'Éducation du Québec (2001). Programme de formation de l'école québécoise. Québec, Québec: Gouvernement du Québec.

Ministère de l'Éducation du Québec (2003). Rapport sur l'évaluation du cheminement particulier de formation visant l'insertion sociale et professionnelle des jeunes de 16 à 18 ans (ISPJ). Québec, Québec: Gouvernement du Québec, Direction de l'adaptation scolaire et des services complémentaires (DASSC).

Ministère de l'Éducation du Québec (2004). Programme de formation de l'école québécoise, enseignement secondaire - $1^{\text {er }}$ cycle. Québec, Québec: Gouvernement du Québec.

Pellerin, G. (2005). Les TIC en classe, une porte ouverte sur la motivation. Québec français, $137,70-72$.

Plouffe, V. (2000). Opinions d'enseignants des programmes adaptés sur des activités pédagogiques inspirées de l'approche d'éducation à la carrière. Mémoire de maîtrise inédit, Université de Sherbrooke, Sherbrooke, Québec.

Potvin, P. (2005). Le transfert de connaissances en sciences sociales et humaines: une valeur ajoutée à la recherche. Table ronde: les conditions de succès du transfert: de multiples défis et un changement de culture. Communication présentée au $73^{\mathrm{e}}$ congrès de l'ACFAS, Université du Québec à Chicoutimi, Saguenay, Québec: Association francophone pour le savoir (ACFAS). [En ligne]. Disponible le 19 février 2008: http://www.ctreq.qc.cal 
88 Revue des sciences de l'éducation, volume 35, n 1, 2009

publications/archives-communiques/colloque-2005/accroitre-transfert-de-connaissance. html

Savoie-Zajc, L. et Lanaris, C. (2005). Regards et réflexions d'une communauté face au problème de l'abandon scolaire. Revue des sciences de l'éducation, 31(2), 297-316.

Viau, R. (1994). La motivation en contexte scolaire. Saint-Laurent, Québec: Éditions du Renouveau Pédagogique.

Viau, R. (1999). La motivation dans l'apprentissage du français. Saint-Laurent, Québec: Éditions du Renouveau Pédagogique.

Vultur, M. (2005). Aux marges de l'insertion sociale et professionnelle. Étude sur les jeunes « désengagés». Nouvelles pratiques sociales, 17(2), 96-108.

Isabelle Landry est actuellement directrice de l'édition, secteur préscolaire et primaire (collection Atouts), chez Septembre éditeur.

\section{Correspondance}

godelieve.debeurme@USherbrooke.ca

isabelle@septembre.com

Ce texte a été révisé par Gilberte Février.

Texte reçu le: 12 décembre 2006

Version finale reçue le: 24 février 2008

Accepté le: 10 mars 2008 
Annexe 1

Exemple de planification : projet Recherche d'emploi

\begin{tabular}{|c|c|c|c|}
\hline Projet: Recherche d'emploi & Parcours: 1- Te connais-tu? & $\begin{array}{l}\text { Étape : A 1-01 Raisons } \\
\text { de chercher un emploi }\end{array}$ & Internet \\
\hline \multicolumn{2}{|c|}{$\begin{array}{l}\text { Domaines généraux de formation: } \\
\text { Orientation et entrepreneuriat: Conscience de soi, de son } \\
\text { potentiel et de ses modes d'actualisation: Connaissance de } \\
\text { ses talents, de ses qualités, de ses intérêts et de ses } \\
\text { aspirations personnelles et professionnelles (Ministère de } \\
\text { l'Éducation du Québec [2004]) }\end{array}$} & \multicolumn{2}{|c|}{$\begin{array}{l}\text { Compétences transversales: } \\
\text { Ordre personnel et social : } 1 \text {. Structurer } \\
\text { son identité: Souvrir aux stimulations } \\
\text { environnantes }\end{array}$} \\
\hline \multicolumn{2}{|c|}{$\begin{array}{l}\text { Compétences disciplinaires: } \\
\text { Lire des textes variés: Utiliser le contenu des textes à } \\
\text { diverses fins }\end{array}$} & \multicolumn{2}{|c|}{$\begin{array}{l}\text { Savoirs essentiels: } \\
\text { Stratégies liées à la gestion et à la } \\
\text { communication de l'information: } \\
\text { Sélectionner des éléments } \\
\text { d'information utiles (réponses aux } \\
\text { questions, nouvelles informations, etc.) }\end{array}$} \\
\hline
\end{tabular}

\section{Objectifs des programmes d'ISPJ - Préparation au marché du travail et insertion sociale}

Préparation au marché du travail : Module 1 : Monde du travail : Unité 6 : Recherche d'emploi :

1.6 Produire le plan d'une démarche personnelle d'entrée sur le marché du travail: 1.6. 3 Décrire les étapes qu'il faudra suivre pour préparer son entrée sur le marché du travail.

Insertion sociale: $2^{e}$ année: Module 4: Besoin essentiel: Réalisation sur le plan personnel: Assumer les formes d'engagement lui permettant de se réaliser sur le plan personnel: Unité 3: Développement personnel : 3.1 Saisir la relation entre la nécessité de bien se connaitre et le fait de définir une orientation générale satisfaisante: 3.1.1 Associer entre eux les éléments de connaissance qu'il a de lui-même

\section{Description de l'étape :}

L'élève coche en cliquant les raisons pour lesquelles il veut travailler et clique sur Continuer

\section{Consignes à l'enseignant:}

Si c'est le premier projet auquel les jeunes participent sur ce site, s'assurer qu'ils sont à l'aise avec l'informatique, les différentes options du site (ex. : carnet de notes, dictionnaire personnel, etc.), les accompagner jusqu'à la prochaine étape pour repérer leurs difficultés

\begin{tabular}{|c|c|c|c|}
\hline Projet: Recherche d'emploi & Parcours: 1- Te connais-tu? & $\begin{array}{l}\text { Étape: A 1-02 Tes } \\
\text { propres raisons de } \\
\text { travailler }\end{array}$ & Internet \\
\hline \multicolumn{2}{|c|}{$\begin{array}{l}\text { Domaines généraux de formation: } \\
\text { Orientation et entrepreneuriat: Conscience de soi, de son } \\
\text { potentiel et de ses modes d'actualisation: Connaissance de } \\
\text { ses talents, de ses qualités, de ses intérêts et de ses } \\
\text { aspirations personnelles et professionnelles }\end{array}$} & \multicolumn{2}{|c|}{$\begin{array}{l}\text { Compétences transversales: } \\
\text { Ordre personnel et social : } 1 \text {. Structurer } \\
\text { son identité : S'ouvrir aux stimulations } \\
\text { environnantes }\end{array}$} \\
\hline \multicolumn{2}{|c|}{$\begin{array}{l}\text { Compétences disciplinaires: } \\
\text { Écrire des textes variés: Exploiter l'écriture à diverses fins }\end{array}$} & \multicolumn{2}{|c|}{$\begin{array}{l}\text { Savoirs essentiels: } \\
\text { Stratégies d'écriture: Stratégies de } \\
\text { mise en texte: Relire la partie rédigée } \\
\text { pour enchaîner la suite }\end{array}$} \\
\hline
\end{tabular}


Annexe 1 (suite)

Exemple de planification : projet Recherche d'emploi

Objectifs des programmes d'ISPJ - Préparation au marché du travail et insertion sociale Préparation au marché du travail : Module 1 : Monde du travail : Unité 6 : Recherche d'emploi : 1.6 Produire le plan d'une démarche personnelle d'entrée sur le marché du travail : 1.6.3 Décrire les étapes qu'il faudra suivre pour préparer son entrée sur le marché du travail

Insertion sociale: $2^{\mathrm{e}}$ année: Module 4: Besoin essentiel: Réalisation sur le plan personnel: Assumer les formes d'engagement lui permettant de se réaliser sur le plan personnel: Unité 3: Développement personnel: 3.1 Saisir la relation entre la nécessité de bien se connaitre et le fait de définir une orientation générale satisfaisante: 3.1.1 Associer entre eux les éléments de connaissance qu'il a de lui-même

\section{Description de l'étape:}

L'élève lit les raisons qu'il a sélectionnées dans l'étape précédente et ajoute ses propres raisons de travailler. Il clique sur Continuer

\section{Consignes à l'enseignant :}

Il peut être intéressant d'animer une discussion de groupe sur d'autres raisons qui les incitent à travailler.

\begin{tabular}{|c|c|c|c|}
\hline Projet: Recherche d'emploi & $\begin{array}{l}\text { Parcours: } 1 \text { - } \\
\text { Te connais-tu? }\end{array}$ & Étape: A1 & Internet \\
\hline \multicolumn{3}{|c|}{$\begin{array}{l}\text { Description de l'étape: } \\
\text { L'élève clique sur le bouton Connais-toi (lien Internet) et fait les activités } \\
\text { proposées dans la section } 1 \text {. Quand il lit, il garde en tête son intention: } \\
\text { mieux se connaître pour trouver un métier qui lui convient vraiment! } \\
\text { Quand il a terminé, il clique sur Terminer }\end{array}$} & Type de rétroaction: \\
\hline \multicolumn{3}{|c|}{$\begin{array}{l}\text { Consignes à l'enseignant: } \\
\text { Cette étape est particulière: c'est la seule où le jeune quitte } \\
\text { l'environnement sécurisé de ce site pédagogique. ll est donc pertinent } \\
\text { de s'assurer qu'il reste sur le site suggéré. Le site présente } 8 \text { activités en } \\
\text { lien avec la connaissance de soi. À vous de juger de la quantité et de la } \\
\text { pertinence de ces activités selon les intérêts et les capacités de vos } \\
\text { élèves }\end{array}$} & $\begin{array}{l}\text { es ou matériel } \\
\text { ers: } \\
\text { let } h \text { ttp ://youth. } \\
\text { aux. } \\
\text { g=fr\&flash }=1 \& t a= \\
\text { geid=199) }\end{array}$ \\
\hline \multicolumn{2}{|c|}{$\begin{array}{l}\text { Domaines généraux de formation: } \\
\text { Orientation et entrepreneuriat: Conscience de soi, } \\
\text { de son potentiel et de ses modes d'actualisation: } \\
\text { Connaissance de ses talents, de ses qualités, de } \\
\text { ses intérêts et de ses aspirations personnelles et } \\
\text { professionnelles }\end{array}$} & \multicolumn{2}{|c|}{$\begin{array}{l}\text { Compétences transversales: } \\
\text { Ordre personnel et social : } 1 \text {. Structurer son } \\
\text { identité: S'ouvrir aux stimulations environnantes }\end{array}$} \\
\hline \multicolumn{2}{|c|}{$\begin{array}{l}\text { Compétences disciplinaires: } \\
\text { Lire des textes variés: Utiliser le contenu des } \\
\text { textes à diverses fins }\end{array}$} & \multicolumn{2}{|c|}{$\begin{array}{l}\text { Savoirs essentiels: } \\
\text { Stratégie de lecture: Stratégies de gestion de la } \\
\text { compréhension } \\
\text { Préciser son intention de lecture et la garder à } \\
\text { l'esprit }\end{array}$} \\
\hline \multicolumn{4}{|c|}{$\begin{array}{l}\text { Objectifs des programmes d'ISPJ - Préparation au marché du travail et insertion sociale } \\
\text { Insertion sociale: module } 4 \text { : Besoin essentiel : Réalisation sur le plan personnel Assumer les formes } \\
\text { d'engagement lui permettant de se réaliser sur le plan personnel: Unité } 3 \text { : Développement } \\
\text { personnel: } 3.1 \text { Saisir la relation entre la nécessité de bien se connaître et le fait de définir une } \\
\text { orientation générale satisfaisante: } 3.1 .1 \text { Associer entre eux les éléments de connaissance qu'il a de } \\
\text { lui-même }\end{array}$} \\
\hline
\end{tabular}


Annexe 2

Exemple de planification : projet de mini-entreprise

\begin{tabular}{|c|c|c|c|}
\hline & & & apier \\
\hline \multicolumn{2}{|c|}{$\begin{array}{l}\text { Domaines généraux de formation: } \\
\text { Orientation et entrepreneuriat: Connaissance du } \\
\text { monde du travail, des rôles sociaux, des métiers et } \\
\text { des professions: } \\
\text { - Lieux de travail (usine, commerces et entreprises de } \\
\text { la région) } \\
\text { - Fonctions principales et conditions d'exercices d'un } \\
\text { emploi }\end{array}$} & \multicolumn{2}{|c|}{$\begin{array}{l}\text { Compétences transversales: } \\
\text { Les compétences d'ordre intellectuel: } \\
\text { 1. exploiter l'information: reconnaître diverses } \\
\text { sources d'information } \\
\text { 3. exercer son jugement critique: construire } \\
\text { son opinion }\end{array}$} \\
\hline \multicolumn{2}{|c|}{$\begin{array}{l}\text { Compétences disciplinaires: } \\
\text { Lire des textes variés: Utiliser les stratégies, les } \\
\text { connaissances et les techniques requises par la } \\
\text { situation de lecture } \\
\text { Communiquer oralement: Explorer verbalement divers } \\
\text { sujets avec autrui pour construire sa pensée }\end{array}$} & \multicolumn{2}{|c|}{$\begin{array}{l}\text { Savoirs essentiels: } \\
\text { Stratégies liées à la gestion et à la } \\
\text { communication de l'information: } \\
\text { - Rencontrer et interroger, s'il y a lieu, des } \\
\text { personnes-ressources } \\
\text { - Sélectionner des éléments d'information } \\
\text { utiles (réponses aux questions, nouvelles } \\
\text { informations, etc.) }\end{array}$} \\
\hline \multicolumn{4}{|c|}{$\begin{array}{l}\text { Objectifs des programmes d'ISPJ - Préparation au marché du travail et insertion sociale } \\
\text { Préparation au marché du travail : } \\
\text { - Module } 1: \text { Monde du travail : L'élève devra développer une connaissance fonctionnelle de } \\
\text { l'organisation du monde du travail : Unité } 1: \text { Milieux de travail : } 1.4 \text { Décrire les principaux milieux de } \\
\text { travail : 1.4.4 Énoncer des exigences particulières d'un milieu de travail } \\
\text { - Module } 2 \text { : Économie: Production et consommation: L'élève devra comprendre qu'il est, lui aussi, } \\
\text { un acteur de la structure socio-économique: Travailleur en tant que producteur : } 2.3 .2 \text { Établir les } \\
\text { liens entre différents types d'acteurs de la structure socio-économique }\end{array}$} \\
\hline \multicolumn{3}{|c|}{$\begin{array}{l}\text { Description de l'étape: } \\
\text { Les élèves visitent une vraie entreprise et répondent à } 5 \text { questions sur leur visite } \\
\text { d'une vraie entreprise qui correspond au genre de mini-entreprise qu'ils veulent } \\
\text { créer }\end{array}$} & \\
\hline \multicolumn{3}{|c|}{$\begin{array}{l}\text { Consignes à l'enseignant: } \\
\text { Pour réaliser cette étape, il serait pertinent de communiquer avec une entreprise } \\
\text { dont le profil correspond à ce que vous désirez créer à l'école. Il serait intéressant } \\
\text { qu'un élève soit chargé d'établir le contact avec l'entreprise pour prendre rendez- } \\
\text { vous pour une visite. Vous pouvez décider de préparer la visite en choisissant par } \\
\text { exemple qui posera les questions lors de la visite } \\
\text { Vous pouvez aussi inviter l'élève à prendre des notes sur toutes les découvertes } \\
\text { qu'il fait }\end{array}$} & \\
\hline
\end{tabular}


Annexe 2 (suite)

Exemple de planification: projet de mini-entreprise

\begin{tabular}{|l|l|}
\hline $\begin{array}{l}\text { Projet: Projet } \\
\text { de mini-entreprise }\end{array}$ & $\begin{array}{l}\text { Parcours : 2- Va voir sur le } \\
\text { terrain comment est organisée } \\
\text { une entreprise }\end{array}$ \\
\hline Domaines généraux de formation: \\
Orientation et entrepreneuriat: \\
- Connaissance du monde du travail, des rôles sociaux, \\
des métiers et des professions \\
- Lieux de travail (usine, commerces et entreprises de la \\
région) \\
- Fonctions principales et conditions d'exercices d'un \\
emploi \\
- Exigences du monde du travail en comparaison avec \\
celles du métier d'élèves \\
- Appropriation des stratégies liées à un projet: \\
Conscience des liens entre connaissance de soi et \\
projets d'avenir
\end{tabular}

Compétences disciplinaires:

Écrire des textes variés:

Utiliser les stratégies, les connaissances et les techniques requises par la situation d'écriture

Recourir à son bagage de connaissances et d'expériences Exploiter l'écriture à diverses fins

\section{Savoirs essentiels :}

Stratégie d'écriture: Stratégies de mise en texte: Ajouter au fur et à mesure les idées qui surviennent

Connaissances liées à la phrase:

- Accords dans la phrase

- Marques de la conjugaison des verbes inclus dans les mots fréquents aux modes et tempos utilisés à l'écrit

- Participe présent et passé

- Sujet/participe passé employé avec l'auxiliaire être

- Reconnaissance et utilisation du groupe du nom : Fonctionnement des accords en genre et en nombre des noms et des adjectifs ( $y$ compris les participes passés employés comme adjectifs)

Objectifs des programmes d'ISPJ - Préparation au marché du travail et insertion sociale Préparation au marché du travail :

- Module 1: Monde du travail : L'élève devra développer une connaissance fonctionnelle de l'organisation du monde du travail: Unité 1 : Milieux de travail : 1.4 Décrire les principaux milieux de travail : 1.4.4 Énoncer des exigences particulières d'un milieu de travail

- Module 2: Économie: Production et consommation: L'élève devra comprendre qu'il est, lui aussi, un acteur de la structure socio-économique: Travailleur en tant que producteur : 2.3.2 Établir les liens entre différents types d'acteurs de la structure socio-économique

\section{Description de l'étape:}

L'élève écrit un texte sur ce que sa visite dans une vraie entreprise lui a apporté. 11 doit utiliser au moins 6 participes passés.

\section{Consignes à l'enseignant :}

Il peut être pertinent de faire une bonne révision des participes passés avant cette activité ou de regarder avec les élèves la théorie présentée lorsqu'ils cliquent sur le bouton Participe passé.

Type de rétroaction: Obligatoire

Ressources ou matériels particuliers : - 
Annexe 3

Questionnaire d'évaluation de Clicfrançais

Version ENSEIGNANT

\begin{tabular}{ccc}
\hline & Totalement Assez Un peu Un peu en Assez en $\begin{array}{c}\text { Totalement } \\
\text { d'accord }\end{array}$ d'accord d'accord désaccord désaccord en désaccord \\
\hline
\end{tabular}

\section{Atteinte des objectifs}

1. Le site répond à un besoin en matériel pédagogique adapté aux caractéristiques des élèves.

2. Le site et son contenu pédagogique sont attrayants, interactifs et dynamiques.

3. Le site contribue à motiver les élèves à l'apprentissage du français.

4. Le site développe des habiletés de préparation et d'intégration au marché du travail chez les élèves.

\section{Niveau de satisfaction}

5. Le contenu est bien adapté aux élèves.

6. Les contenus d'apprentissage proposés sont pertinents et de qualité.

7. Le guide d'accompagnement appuie adéquatement les activités du site et la démarche pédagogique proposée.

8. Les projets s'insèrent bien dans le contexte de la classe, dans le respect des objectifs du MÉLS et du temps accordé pour atteindre les compétences visées.

9. Il est simple d'utiliser les multiples fonctionnalités du site (messagerie, forums, carnet de notes, révision et publication des travaux, etc.).

10. Il est facile de naviguer dans le site.

11. Le concept visuel du site est attrayant, agréable et harmonieux.

12. Dans votre école, vous disposez de l'équipement informatique et de la configuration requis pour profiter pleinement du site.

13. De façon générale, vous êtes satisfait du site (intérêt, utilité, originalité). 
Questionnaire d'évaluation de Clicfrançais

Version ENSEIGNANT

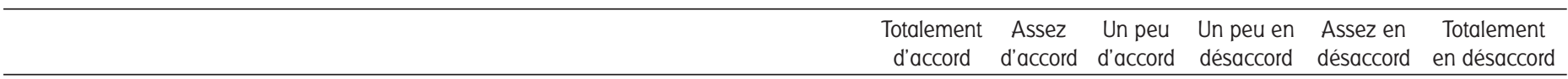

\section{Atteinte des objectifs}

\section{Impacts de l'outil}

14. Le site est un apport significatif à l'amélioration ou au renouvellement des pratiques

d'enseignement en ISPJ.

15. L'utilisation de cet outil pour l'enseignement du français a fait hausser le taux de motivation pour son apprentissage chez vos élèves.

16. Les projets proposés dans le site ont suscité beaucoup d'intérêt chez vos élèves.

Questionnaire d'évaluation de Clicfrançais (suite)

version ENSEIGNANT -

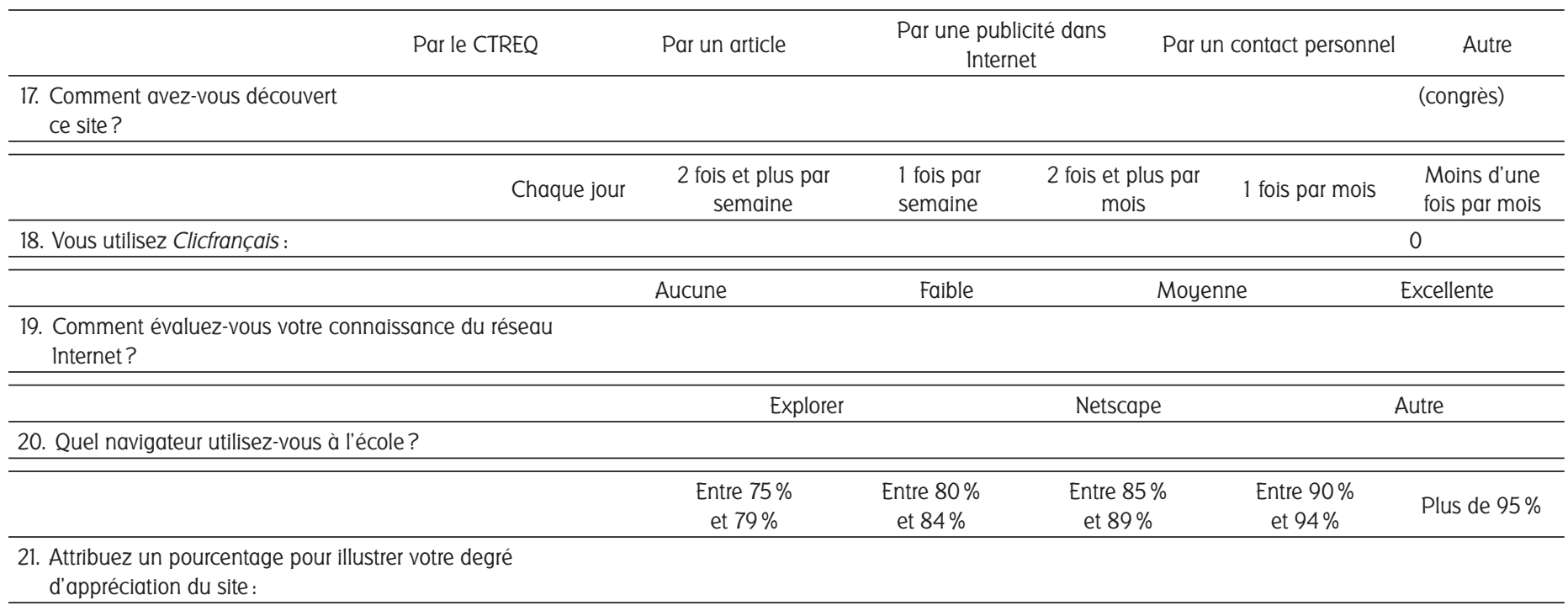


Questionnaire d'évaluation de Clicfrançais

Version ÉLĖVE

\begin{tabular}{cc}
\hline Assez & Assez en \\
désaccord & $\begin{array}{c}\text { Totalement } \\
\text { en désaccord }\end{array}$ \\
d'accord & d'accord
\end{tabular}

\section{Atteinte des objectifs}

1. Les activités proposées dans Clicfrançais me plaisent, elles sont dynamiques et interactives.

2. J'aime réaliser des projets à l'ordinateur.

3. Je me sens plus motivé(e) à apprendre quand j'utilise Clicfrançais.

4. Les activités présentées dans Clicfrançais me permettent d'être mieux préparé(e) pour mon futur emploi.

\section{Niveau de satisfaction}

5. La recherche d'emploi et la création d'une entreprise sont des sujets qui m'intéressent.

6. Je crois que les projets Recherche d'emploi et Mini-entreprise sont utiles.

7. J'ai trouvé les étapes du projet faciles à réaliser.

8. J'aime les couleurs du site et les illustrations.

9. Le site et ses outils (carnet de notes, messagerie, forums, dictionnaire personnel, etc.) sont simples à utiliser.

10. Les textes sont faciles à lire et je les comprends bien.

11. Il est facile de naviguer dans Clicfrançais.

12. De façon générale, j'aime Clicfrançais.

\section{Impacts de Clicfrançais}

13. Depuis que je participe à Clicfrançais, j'ai amélioré mes connaissances et mes habiletés en français.

14. Depuis que je participe à Clicfrançais, j'ai augmenté mes connaissances sur le monde du travail (ses exigences, ses possibilités...).

15. Depuis que je participe à Clicfrançais, je me connais mieux (mes intérêts, mes compétences, mes habiletés d'employabilité, etc.).

16. J'ai le goût de faire des activités en français dans Clicfrançais.

17. Je vais souvent au local d'informatique de mon école. 
Version ÉLÈVE

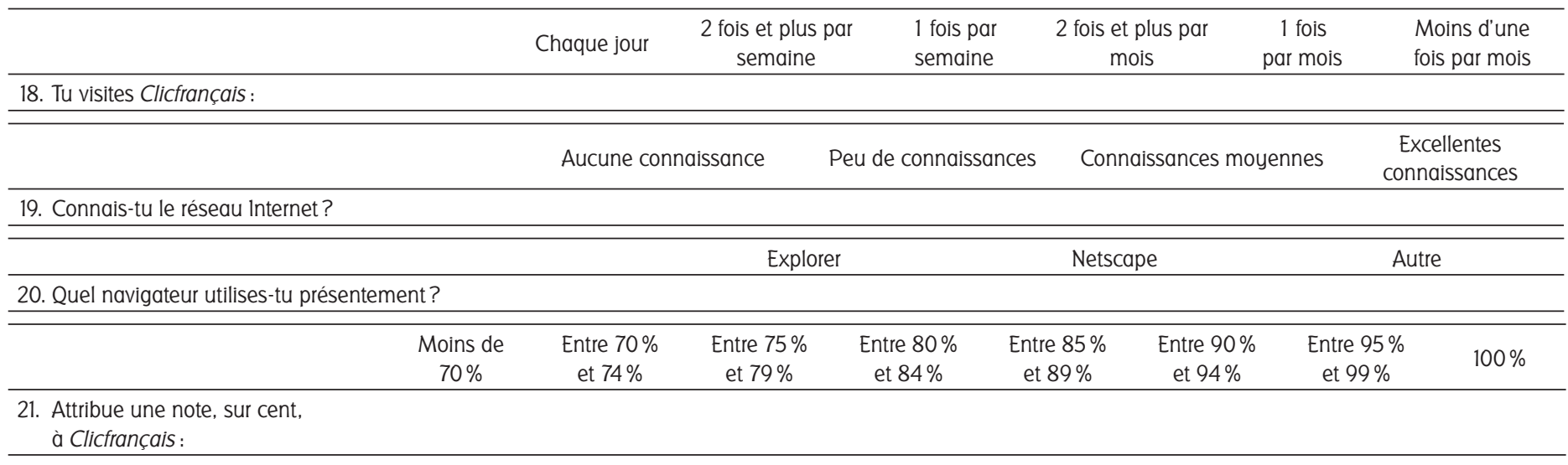


Grille: Critères et indicateurs utilisés pour l'évaluation

\begin{tabular}{|c|c|c|}
\hline Critères & & Indicateurs \\
\hline \multicolumn{3}{|l|}{ Pertinence } \\
\hline \multirow{6}{*}{$\begin{array}{l}\text { 1. Réponse } \\
\text { à un besoin } \\
\text { de la clientèle }\end{array}$} & - & L'analyse du besoin à l'origine du site était de qualité \\
\hline & $\bullet$ & $\begin{array}{l}\text { Les résultats de recherche faisant l'objet du transfert avaient un potentiel } \\
\text { d'application }\end{array}$ \\
\hline & - & Les objectifs du site étaient clairs \\
\hline & 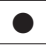 & Les clientèles visées par le site ont été identifiées adéquatement \\
\hline & - & Le plan de diffusion du site était de qualité \\
\hline & 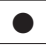 & Le site est accessible (du point de vue économique et technologique) \\
\hline \multirow{4}{*}{$\begin{array}{l}\text { 2. Concept } \\
\text { du produit }\end{array}$} & ○ & Le concept du site était bien décrit \\
\hline & ○ & Il y a correspondance entre les objectifs du devis et ceux du concept du site \\
\hline & ? & Il y a correspondance entre le concept et le produit réalisé \\
\hline & $\bullet$ & Le site a une durée de vie (pérennité) appropriée \\
\hline \multicolumn{3}{|l|}{ Efficacité } \\
\hline \multirow{2}{*}{$\begin{array}{l}\text { 3. Atteinte } \\
\text { des objectifs }\end{array}$} & $\bullet$ & $\begin{array}{l}\text { La réalisation du site est en réponse à un besoin exprimé par les } \\
\text { enseignants }\end{array}$ \\
\hline & $\bullet$ & $\begin{array}{l}\begin{array}{l}\text { Les objectifs visés par le site sont atteints pour les clientèles (enseignants et } \\
\text { élèves) }\end{array}\end{array}$ \\
\hline \multirow{5}{*}{$\begin{array}{l}\text { 4. Satisfaction } \\
\text { de la clientèle }\end{array}$} & $\bullet$ & $\begin{array}{l}\text { Le site, dans son ensemble, est satisfaisant pour les clientèles (enseignants } \\
\text { et élèves) }\end{array}$ \\
\hline & $\bullet$ & $\begin{array}{l}\text { L'ergonomie et de la convivialité du site sont satisfaisants pour les clientèles } \\
\text { (enseignants et élèves) }\end{array}$ \\
\hline & $\bullet$ & $\begin{array}{l}\text { Le niveau de langage utilisé dans le site est satisfaisant pour les clientèles } \\
\text { (enseignants et élèves) }\end{array}$ \\
\hline & $\bullet$ & $\begin{array}{l}\text { L'adaptation du contenu du site est satisfaisante pour les clientèles } \\
\text { (enseignants et élèves) }\end{array}$ \\
\hline & $\bullet$ & $\begin{array}{l}\text { La qualité visuelle du site est satisfaisante pour les clientèles (enseignants et } \\
\text { élèves) }\end{array}$ \\
\hline \multirow{6}{*}{$\begin{array}{l}\text { 5. Diffusion du } \\
\text { produit }\end{array}$} & - & $\begin{array}{l}\text { Les moyens mis en œuvre pour la diffusion et la promotion du site sont } \\
\text { efficaces }\end{array}$ \\
\hline & $\bullet$ & $\begin{array}{l}\text { Le nombre de canaux utilisés pour la diffusion et la promotion du site est } \\
\text { adéquat }\end{array}$ \\
\hline & $\bullet$ & $\begin{array}{l}\text { Le nombre d'utilisateurs du site rejoints par rapport au nombre visé ou } \\
\text { potentiel est adéquat }\end{array}$ \\
\hline & 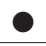 & La durée de vie du site est adéquate \\
\hline & $\bullet$ & Le nombre de partenaires (et leur rôle) est adéquat \\
\hline & ? & Les ressources investies par les partenaires sont suffisantes \\
\hline \multirow{3}{*}{$\begin{array}{l}\text { 6. Ressources } \\
\text { investies dans } \\
\text { le produit }\end{array}$} & ? & $\begin{array}{l}\text { Le budget pour la conception, la réalisation, la diffusion et l'évaluation est } \\
\text { respecté }\end{array}$ \\
\hline & O & Les efforts requis (en ressources humaines) sont adéquats \\
\hline & O & L'ampleur des ressources informationnelles et techniques est adéquate \\
\hline
\end{tabular}


98 Revue des sciences de l'éducation, volume 35, n 1, 2009

Grille: Critères et indicateurs utilisés pour l'évaluation (suite)

\begin{tabular}{|c|c|l|}
\hline Impact & & \\
\hline \multirow{4}{*}{$\begin{array}{c}\text { 7. Impacts } \\
\text { du produit }\end{array}$} & $\bullet$ & $\begin{array}{l}\text { La proportion utilisateurs du site / utilisateurs rejoints est satisfaisante chez } \\
\text { les clientèles (enseignants et élèves) }\end{array}$ \\
\cline { 2 - 3 } & $\bullet$ & $\begin{array}{l}\text { La fréquentation du site est satisfaisante en ce qui concerne les clientèles } \\
\text { (enseignants et élèves) }\end{array}$ \\
\cline { 2 - 3 } & - & $\begin{array}{l}\text { Grâce au site, on estime avoir amélioré ses façons de faire chez les clientèles } \\
\text { (enseignants et élèves) }\end{array}$ \\
\cline { 2 - 3 } & - & $\begin{array}{l}\text { De nouvelles collaborations avec des partenaires (actuels ou non) ont été } \\
\text { créées }\end{array}$ \\
\hline $\begin{array}{c}\text { 8. Impacts hors } \\
\text { cibles }\end{array}$ & - & Le site a généré des retombées non anticipées \\
\hline
\end{tabular}

Légende

- Indicateur retenu avec informations suffisantes

Indicateur retenu avec informations insuffisantes 